\title{
Spatially Shaped Laser Pulses for the Simultaneous Detection of Polycyclic Aromatic Hydrocarbons as well as Positive and Negative Inorganic Ions in Single Particle Mass Spectrometry
}

Julian Schade + , Johannes Passig* ${ }^{* \dagger}$, Robert Irsig" ${ }^{\|}$, Sven Ehlert"l, Martin Sklorz ${ }^{\ddagger}$, Thomas Adams, Chunlin $\mathrm{Li}^{+}$, Yinon Rudich ${ }^{+}$and Ralf Zimmermann ${ }^{\ddagger}$

† Joint Mass Spectrometry Centre, Chair of Analytical Chemistry, University Rostock, 18059 Rostock, Germany ‡Joint Mass Spectrometry Centre, Cooperation Group ‘Comprehensive Molecular Analytics’ (CMA), Helmholtz Zentrum München, 85764 Neuherberg, Germany

"Photonion GmbH, 19061 Schwerin, Germany

§ Bundeswehr University Munich, 85577 Neubiberg, Germany

${ }$ Department of Earth and Planetary Sciences, Weizmann Institute of Science, Rehovot 76100, Israel

*corresponding author: johannes.passig@uni-rostock.de

\section{Content:}

Figure S1:

Schematic view of an alternative optical setup for the combined LDI/REMPI ionization step.

Figure S2:

Mass spectra of an exemplary organic particle that was missed by the laser desorption and thus shows strong fragmentation from direct LDI.

Figure S3:

Particle size distribution measured in the ambient air experiment.

Figure S4:

Particle size distributions of the model particles. 


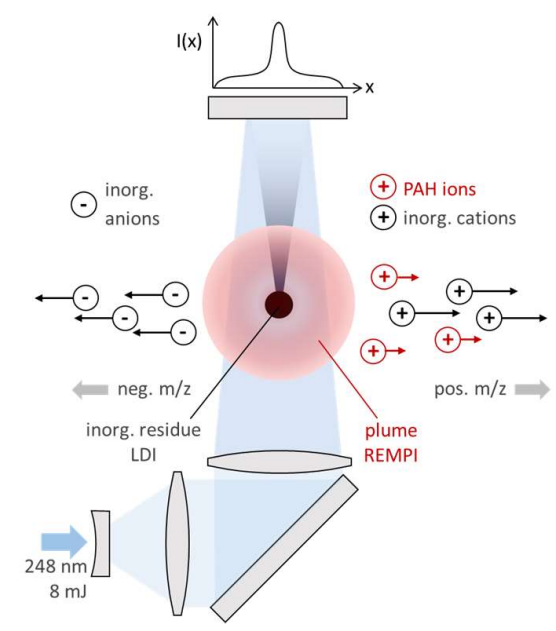

Figure S1. Schematic view of an alternative optical setup for the combined LDI/REMPI ionization step (previous laser desorption not shown). Using a lens with a long focal length and a planar mirror for back-reflection allows the independent adjustment of the REMPI beam width and intensity.

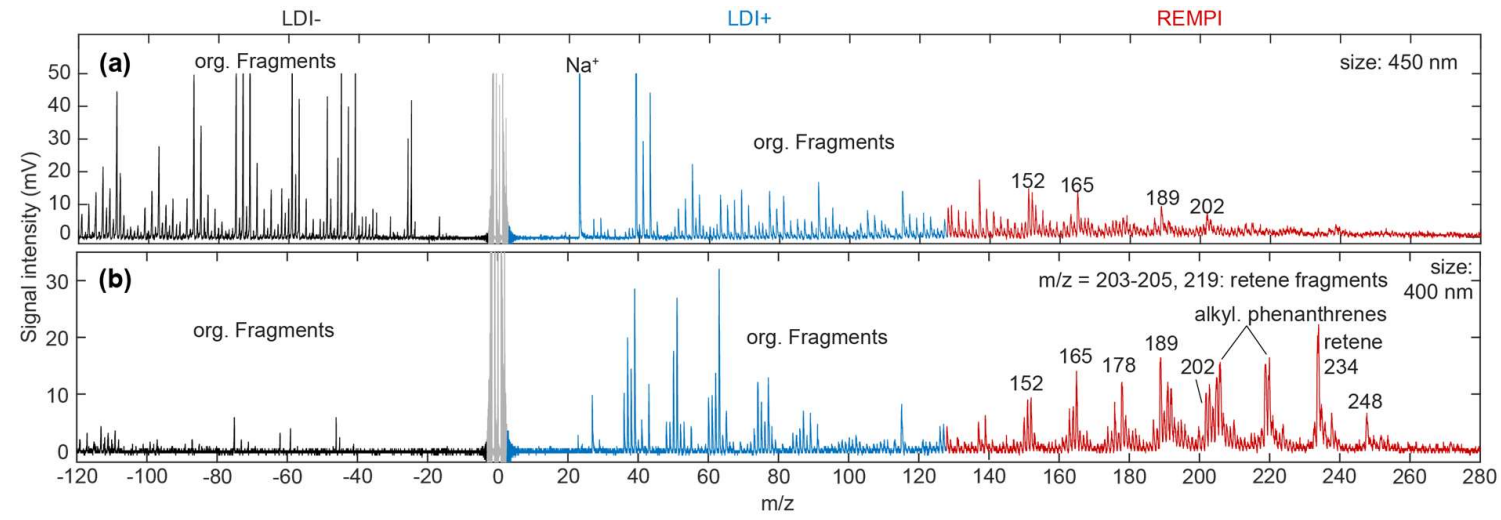

Figure S2. (a) Exemplary spectra of a tar ball particle that was not exposed to a laser desorption pulse but hit by the intense LDI beam, comparable to conventional SPMS. The spectrum exhibits only fragments and sodium. The strong signals from anions and $\mathrm{Na}^{+}$result from the dense plume upon LDI. (b) For comparison, the tar ball spectrum already shown in Figure 2(c) illustrates the superposition of LDI and LD-REMPI utilized in our method. 

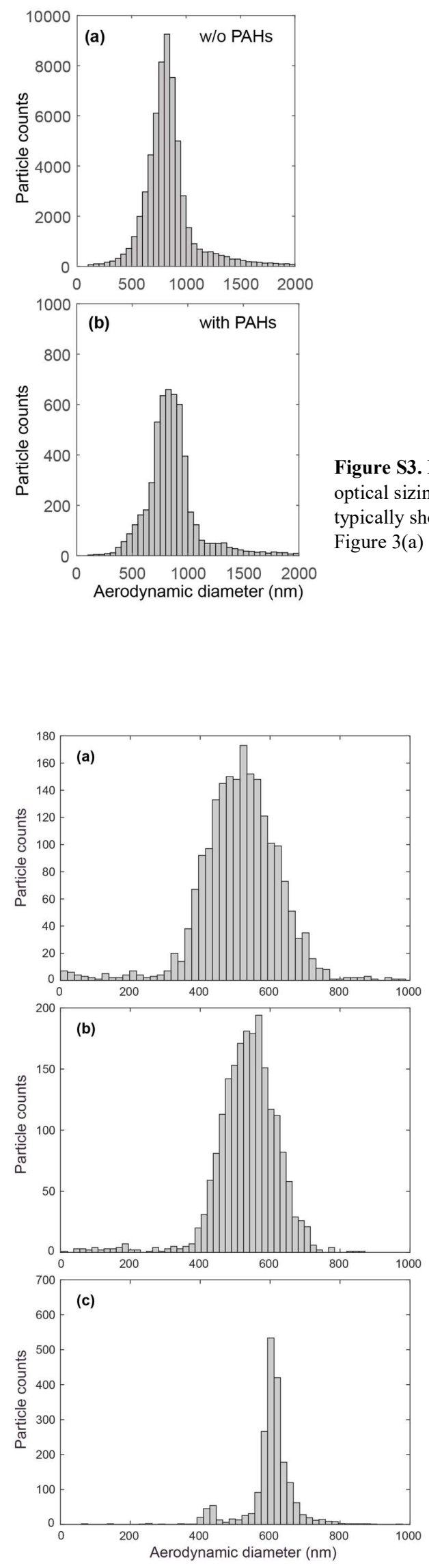

Figure S3. Particle size distribution in the ambient air experiment, measured with the optical sizing unit of the SPMS instrument. Note that long-range transported particles typically show a narrow distribution. (a) Particles without PAHs corresponding to Figure 3(a) and (b) particles showing PAH signatures corresponding to Figure 3(b).
Figure S4. Particle size distribution of the model particles, measured with the optical sizing unit of the SPMS instrument. (a) Diesel soot particles corresponding to Figure 2(a), (b) wood ash particles corresponding to Figure 2(b) and (c) tar ball particles corresponding to Figure 2(c). Each $n=2000$ particles, respectively. 